\title{
ORIGINAL
}

\section{REACTIVIDAD CARDIOVASCULAR Y FACTORES DE RIESGO CARDIOVASCULAR EN INDIVIDUOS NORMOTENSOS DEL MUNICIPIO DE RODAS, CIENFUEGOS} \author{
Yanes Nuñez (4) y Manuel Fernández Urquizar (5) \\ (1) Fisiología Facultad de Ciencias Médicas de Cienfuegos \\ (2) Facultad de Ciencias Médicas de Cienfuegos \\ (3) (4) Especialista de primer grado en Medicina General Integral. \\ (5) Neonatología. Facultad de Ciencias Médicas de Cienfuegos.
}

Mikhail Benet Rodríguez (1), Juan J Apollinaire Pinnini (2), Julio González Leiva (3), Aldo Jesús

\section{RESUMEN}

Fundamento: Existe asociación entre diferentes factores de riesgo cardiovasculares con el incremento significativo de la tensión arterial en presencia de estrés psíquico o físico (reactividad cardiovascular). Nosotros estudiamos esta asociación en individuos normotensos sometidos a un estrés provocado por el ejercicio isométrico que nos aporla la prueba del peso sostenido.

Métodos: Se realizó un estudio descriptivo en el que se formaron dos grupos a partir de 267 individuos normotensos. Uno de estudio, de sujetos hiperreactivos cardiovasculares (HRCV) con mayor respuesta de presión arterial $(\mathrm{TA})(\mathrm{n}=58)$, con niveles de TA $\geq 90 / 140 \mathrm{~mm} / \mathrm{Hg}$, y el otro grupo control, con TA $90 / 140 \mathrm{~mm} / \mathrm{Hg}, \mathrm{n}=209$. Se determinó la asociación entre la edad, sexo, sedentarismo, hábito de fumar (HF), ingestión de bebidas alcohólicas (IBA) y los antecedentes patológicos de hipertensión arterial (APF de HTA), con el estado de hiperreactividad cardiovascular

Resultados: el 21.7\% de los individuos fueron HRCV. Los factores de riesgos HF, IBA y sexo no se asociaron con la HRCV P > 0,05. El riesgo de los individuos sedentarios de ser HRCV es 2 veces mayor; la edad se asoció a partir de los 40 años y los individuos con APF de HTA tuvieron un riesgo de tener una hiperreactividad cardiovascular $3.85(2 ; 7,19)$ veces mayor que los que no tenían APF de HTA.

Conclusiones: Existe una asociación significativa entre la edad mayor de 40 años, los APF de HTA y el sedentarismo, con el estado de HRCV. No asi otros factores de riesgo como HF, sexo e IRA

Palabras claves: Enfermedades cardiovasculares. Hipertensión arterial. Ejercicio físico isométrico. Factores de riesgo cardiovasculares.

Correspondencia:

Dr. Mikhail Benet Rodríguez

Dirección: Calatrava 4 5to A CP: 47011 . Valladolid.

Correo electrónico: mmolina $@$ dali.eis.uva.cs

\section{ABSTRACT \\ Cardiovascular Reactivity and Cardiovascular Risks Factors in Normotense Individuals in the Municipality of Rodas, Cienfuegos, Cuba}

Background: A relationship exists between different cardiovascular risk factors and a significant rise in blood pressure in the presence of psychological or physical stress (cardiovascular reactivity). We studied this relationship in nomotense individuals who were subjected to stress caused by isometric exercise, which provided us with proof of the amount of weight withstood.

Methods: A descriptive study was conducted in which 267 normotense individuals were divided into two groups. One of these groups was a study group comprised of cardiovascular hyperreactive (CVHR) with a greater blood pressure response $(\mathrm{BP})(\mathrm{n}=58)$, with BP levels $\geq 90 / 140 \mathrm{~mm} / \mathrm{Hg}$, and the other group as a control group, with $\mathrm{BP}<90 / 140 \mathrm{~mm} / \mathrm{Hg}, \mathrm{n}=209$. The relationship was found between the age, gender, sedentarism, smoking habit (SH), alcoholic beverage intake (ABI) and clinical history of blood pressure disorders (CH of BPD with the condition of cardiovascular hyperreactivity.

Results: $21.7 \%$ of the subjects were CVHR. The risk factors $\mathrm{SH}, \mathrm{ABI}$ and gender were not related to the CVHR P $>$ 0.05 . The risk factor of CVHR is twice as high on the part of sedentary individuals, age becoming a factor as of age 40 , and those individuals with a $\mathrm{CH}$ of BPD had twice the risk of having a cardiovascular hyperreactivity $3.85(2: 7.19)$ as those who had no $\mathrm{CH}$ of $\mathrm{BPD}$ ).

Conclusions: A significant relationship exists between being over age $40, \mathrm{CH}$ of BPD and lack of exercise with a CVHR condition. This was not found to be the case for other risk factors such as $\mathrm{SH}$. gender and $\mathrm{ABI}$.

Key words: High blood pressure. Isometric physical exercise. Cardiovascular risk factors. Tobacco. 


\section{INTRODUCCIÓN}

Tanto en la literatura científica como en la clínica, existe desacuerdo sobre la utilidad de las pruebas de ejercicios para predecir el futuro de la hipertensión arterial (HTA) y de las enfermedades cardiovasculares (ECV), así como sobre la relación del estado de hiperreactividad cardiovascular ( $\mathrm{HRCV}$ ) con factores de riesgos cardiovasculares (FRCV) ${ }^{1,2}$, lo que justifica en gran medida la realización de este estudio.

La reactividad cardiovascular ( $\mathrm{RCV}$ ) es definida como los cambios en la presión de la sangre, frecuencia cardiaca u otros parámetros hemodinámicos en respuesta a un estímulo físico o mental ${ }^{2}$. Se ha podido observar que los individuos hipertensos presentan una mayor RCV que los normotensos $\mathrm{y}$, así mismo, se ha encontrado una mayor $\mathrm{RCV}$ en jóvenes que presentan FRCV ${ }^{3}$.

La RCV como respuesta al estrés ha sido propuesta como un factor de riesgo (FR) para la $\mathrm{HTA}^{4-6}$. Por otro lado, se ha evidenciado un aumento de la presión de la sangre (TA), así como de la resistencia periférica total (RPT), en niños con historia familiar de enfermedades de las arterias coronarias con respecto a otros niños sometidos a un estrés físico ${ }^{6}$.

Las técnicas para inducir RCV son varias, entre ellas encontramos el ejercicio físico isométrico y el ejercicio físico isotónico (cargas físicas), el aumento de la presión de $\mathrm{CO} 2$ en la sangre, los cambios ortostáticos, el estímulo del frío y la respuesta presora a diferentes tipos de estrés mental. Se ha observado que las cargas físicas gozan de aceptación unánime, y han demostrado ser mucho más sensibles y específicas que todas las restantes ${ }^{7.8}$.

El método que utilizamos para desarrollar este trabajo se denomina La Prueba del Peso Sostenido (PPS), una variante de las pruebas que utilizan el ejercicio isométrico para inducir modificaciones hemodinámicas del sistema cardiovascular ${ }^{7}$. En la misma se realiza un ejercicio isométrico, manteniendo un peso de 500 gramos en la mano con el brazo izquierdo en ángulo recto con el cuerpo, durante 2 minutos. La presión arterial se toma en el brazo contrario antes del ejercicio y en los últimos 20 segundos del segundo minuto de la misma.

Dada la importancia que tiene conocer en detalle cuáles de los factores de riesgo, pueden contribuir a desencadenar en los individuos normotensos una enfermedad hipertensiva, para desarrollar políticas de salud que modifiquen estilos de vidas poco saludables, se enfocó la atención a determinar la asociación entre algunos de los FRCV más importantes y el estado de respuesta cardiovascular excesiva o hiperreactividad cardiovascular, inducida mediante el ejercicio isométrico, en personas normotensas, y de esta forma consolidar resultados que alrededor de este tema se presentan en la literatura internacional.

\section{MATERIAL Y MÉTODO}

Se realizó un estudio comparativo representativo, en el que se formaron dos grupos a partir de una muestra compuesta por 267 individuos normotensos entre 18 y 70 años de edad, de ambos sexos, todos del consultorio 12 del municipio Rodas, provincia de Cienfuegos, Cuba. Se aplicó la PPS a todas las personas, y se obtuvo la distribución de valores de las presiones sistólica (PS) y diastólica (PD) mediante este método.

Uno de los grupos estuvo formado por los (HRCV), grupo de estudio que incluía a todas las personas normotensas ( 33 mujeres y 25 hombres), con valores de TA después de la PPS igual o superior a $140 / 90 \mathrm{~mm} / \mathrm{Hg}$, y el otro, por los sujetos normorreactivos vasculares (NRCV), grupo control que englobaba a todas las personas normotensas (115 mujeres y 94 hombres), con valores de la TA inferior a $140 / 90 \mathrm{~mm} / \mathrm{Hg}$ después de la PPS. No entraron en el estudio las personas con cardiopatía isquémica y las mujeres em- 
barazadas. Los individuos hipertensos se descartaron mediante los critcrios del Comité Norteamericano para el Control, Evaluación y Seguimiento de la HTA ?

Las variables independientes estudiadas fueron: el sexo, la edad, los antecedentes patológicos familiares de HTA (APF de HTA), el sedentarismo, el peso corporal (PC), el hábito de fumar (HF) y la ingestión de bebidas alcohólicas (IBA). La variable dependiente fue el estado o no de reactividad cardiovascular.

Se consideró como un individuo sedentario a aquel que declaró no realizar ejercicios físicos por lo menos 3 veces por semana durante 30 minutos o más. Los datos de la ingestión de bebidas alcohólicas fueron recogidos atendiendo al criterio de:

— Nunca: no ingiere bebidas alcohólicas.

- Ocasional:1 a 6 veces por año.

- Moderado: 1 a 3 veces por semana $<100 \mathrm{ml}$.

- Excesivo: 1 a 3 veces por semana $\geq 100 \mathrm{ml}$.

- Diario.

Teniendo en cuenta que $100 \mathrm{ml}$ de alcohol equivalen aproximadamente a:

$-1 / 2$ litro de ron.

-1 litro de vino.

- 7 botellas de cerveza.

Esta variable se evaluó agrupando a los individuos en tres categorías: en la primera los que nunca ingirieren bebidas alcohólicas y los que lo hacen ocasionalmente, en la segunda a los que lo hacen moderadamente y en la tercera a los que ingieren excesivamente o diariamente.
Se consideró una persona con APF de IITA cuando el padre, la madre o ambos eran hipertensos.

En relación con cl HF, se tuvo en cuenta el número de cigarros que fumaban diariamente y se agruparon en: no fumador, fumador de menos de 10 cigarrillos diarios, fumador de entre 10 a 19 cigarrillos diarios, fumador de 20 o más cigarrillos diarios.

Para el análisis estadístico de la información se utilizaron los programas SPSS $8.0 \mathrm{y}$ EPIINFO 6, dispuestos en el Laboratorio de Computación de la Facultad de Ciencias Médicas de Cienfuegos. Se obtuvieron los percentiles de la distribución mediante el método de Haverage. La Odds Ratio y el intervalo de confianza se utilizaron para determinar el riesgo de hiperreactividad vascular en presencia de sedentarismo, edad y APF de HTA. Se utilizó la prueba de $\chi^{2}$ para analizar el HF e IBA. El análisis de varianza de un factor y el test de students se usaron para contrastar la hipótesis de diferencias entrc las diferentes categorías de fumadores y la influencia del sexo respectivamente. En todos los casos el nivel de significación que se fijó fue del $95 \%$.

\section{RESULTADOS}

El valor de presión para el 90 percentil de la distribución fue de $140 \mathrm{~mm} / \mathrm{Hg}$ para la PS y de $90 \mathrm{~mm} / \mathrm{Hg}$ para la PD (tabla 1). 209 personas fueron clasificadas como normorreactivas vasculares $(78.3 \%)$ y 58 como reactivas vasculares $(21.7 \%$ ) (figura 1 ).

No se presentaron diferencias importantes entre hiperreactivos y normorreactivos en relación con el número de cigarros que fumaban diariamente (tabla 2). Los niveles de TA entrc las diferentes categorías de fumadores ajustadas por sexo, tampoco evidenció diferencias significativas $\mathrm{P}>0,05$, manteniéndose aproximadamente en $80 \pm 5$ $\mathrm{mm} / \mathrm{Hg}$ para la diastólica y de $130 \pm 5$ $\mathrm{mm} / \mathrm{Hg}$ para la sistólica (tablas 3 y 4 ). 
Tabla 1

Valores medios, desviación estándar e intervalos de normalidad y HTA de los percentiles 10 y 90 para los individuos previamente hipertensos y normotensos. $\mathrm{N}=267$. Rodas, 1998

\begin{tabular}{|c|c|c|c|c|c|c|c|c|}
\hline \multirow{2}{*}{ Previamente } & \multicolumn{4}{|c|}{ Presión Sistólica } & \multicolumn{4}{|c|}{ Presión diastólica } \\
\hline & $X P_{S}$ & Dvs Std & loperc. & 90 Perc & $X P d$ & Dvs Std & loperc. & 9opere \\
\hline Normotensos & $123.2 *$ & 14.6 & 103.3 & 140 & $79.05^{*}$ & 8.92 & 66.6 & 90 \\
\hline
\end{tabular}

Los valores en negritas son los límites tomados para determinar los hipertensos y los no hipertensos.

Figura 1

Frecuencia y por cientos de los individuos normotensos, e hiperreactivos tomando como criterio el 90 percentil de la distribución de presiones de los individuos normotensos que se obtuvo con la PPS. $N=267$. Rodas, 1998

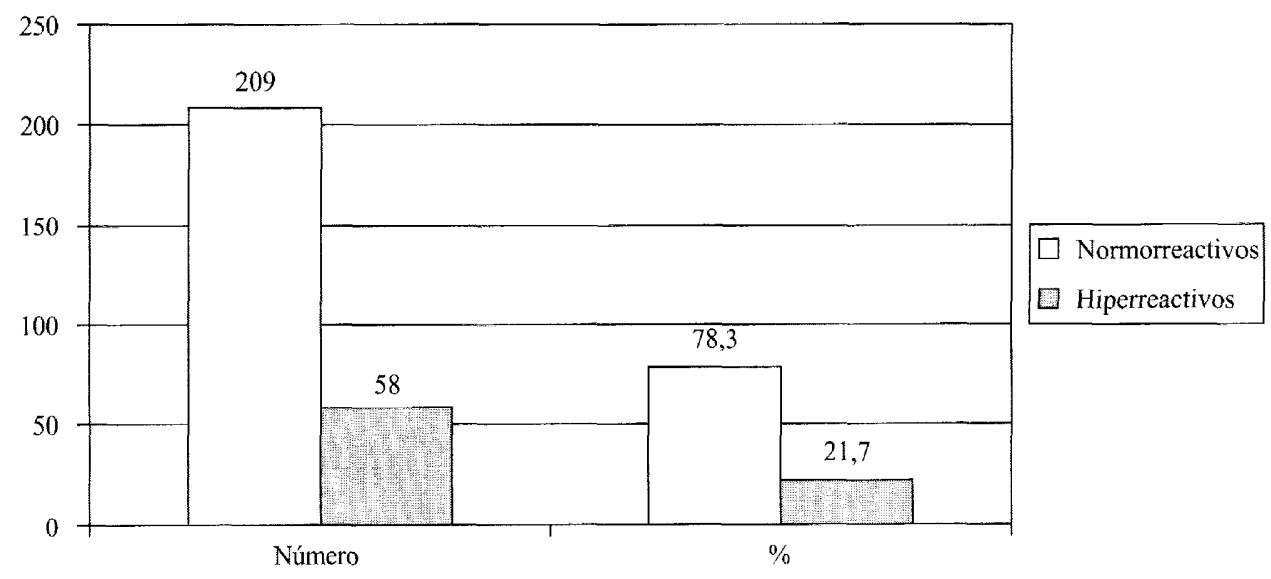

Tabla 2

Comparación entre individuos normorreactivos e hiperreactivos atendiendo al número de cigarros que fuman diariamente. $\mathrm{N}=267$. Rodas, 1998

\begin{tabular}{|c|c|c|c|c|c|c|c|c|c|}
\hline \multirow{2}{*}{ Estado } & \multirow{2}{*}{$N$} & \multicolumn{2}{|c|}{0 cigarros } & \multicolumn{2}{|c|}{ 1.9 cigarros } & \multirow{2}{*}{$\begin{array}{c}\begin{array}{c}10-19 \mathrm{ci}- \\
\text { garros }\end{array} \\
\text { No }\end{array}$} & \multicolumn{2}{|c|}{ + Más de 20} & \multirow[b]{2}{*}{$\%$} \\
\hline & & No & $\%$ & No & $\%$ & & $\%$ & No & \\
\hline Hiperr. & 58 & 43 & 74.1 & 2 & 3.4 & 5 & 8.6 & 8 & 13.8 \\
\hline Nomorr. & 209 & 147 & 70.3 & 8 & 3.8 & 18 & 8.6 & 36 & 17.2 \\
\hline
\end{tabular}

$\chi 2=0,43419 \quad \mathrm{P}=0,93309$ 
Tabla 3

Comparación de los promedios de valores de la TA diastólica obtenidos mediante la PPS para los hombres y la mujeres tomando en cuenta el número de cigarrillos consumidos diariamente. $\mathrm{N}=267$. Rodas, 1998

\begin{tabular}{|lccc|}
\hline$N^{2}$ de cigarros & $X$ de TA hombres & X de TA mujeres & Valor P por filas \\
\hline No fuma & 87.46 & 83.24 & 0.090 \\
De 1 a 9 & 77.33 & 85.03 & 0.280 \\
De $10-19$ & 82.13 & 80.10 & 0.611 \\
Más de 20 & 85.92 & 83.32 & 0.399 \\
Valor P por Columna & 0.4881 & 0.2987 & \\
\hline
\end{tabular}

Tabla 4

Comparación de los promedios de valores de la TA sistólica obtenidos mediante la PPS para los hombres y la mujeres tomando en cuenta el número de cigarrillos consumidos diariamente. $\mathbf{N}=\mathbf{2 6 7}$. Rodas, 1998

\begin{tabular}{|lccc|}
\hline$N^{\circ}$ de cigarros & $X$ de TA hombres & X de TA mujeres & Valor P por filas \\
\hline No fuma & 135.16 & 134.29 & 0.845 \\
De 1 a 9 & 129.33 & 131.77 & 0.784 \\
De $10-19$ & 126.66 & 122.53 & 0.593 \\
Más de 20 & 135.09 & 135.60 & 0.929 \\
Valor P por Columna & 0.5863 & 0.4186 & \\
\hline
\end{tabular}

El valor $\mathrm{P}$ por columna se determinó mediante un análisis de varianza ANOVA de un factor donde se compararon todos los grupos. El valor $\mathrm{P}$ por fillas se determinó mediantc una $\mathrm{T}$ de students para mucstras independientes.

Para los sedentarios la Odds Ratio de HRCV fue de 2 IC (1.04-3.86) respecto a los no sedentarios, y para los que tenían APF de HTA de 3.85 IC $(2.00,7.19)$ con relación a los que no tenían, no hubo diferencias en cuanto al sexo (tabla 5). La edad estuvo asociada al estado de HRCV a partir de los 40 años (tabla 6), donde el número de personas en el grupo de edades de 40-49 fue de $30.4 \%$ y en los grupos $50-59$ y 60 y más, de $28.1 \%$ y $30 \%$ respectivamente.

Tabla 5

Factores de riesgo cardiovascular e hiperreactividad cardiovascular. $\mathrm{N}=267$. Rodas, 1998

\begin{tabular}{|lll|}
\hline \multicolumn{1}{|c}{ Factores de riesgo } & OR & IC 95\% \\
\hline Sedentarismo & 2 & $1.04,3.86$ \\
Sexo & 1.16 & $0.61,2.21$ \\
Antecedentes familiares de HTA & 3.85 & $2.00,7.19$ \\
\hline
\end{tabular}

El 35\% de los individuos que ingerian alcohol diariamente o excesivamente eran HRCV por un $19 \%$ del resto (tabla 7 ).

\section{DISCUSIÓN}

Los criterios para considerar un individuo HRCV son muy variados y no sólo por las numerosas pruebas que se usan para llegar a diagnosticarles como tal, sino también por los niveles de presión que han sido utilizados por otros autores ${ }^{1,7}$. Hiram ${ }^{7}$, con la PPS ha considerado HRCV a las mujeres que tienen niveles de presión arterial media (PAM) superior a $105 \mathrm{~mm} / \mathrm{Hg}$ e inferior a 113 $\mathrm{mm} / \mathrm{Hg}$ y en el caso de los hombres, cuando los niveles de presión están entre 109 $\mathrm{mm} / \mathrm{Hg}$ y $117 \mathrm{~mm} / \mathrm{Hg}$.

El criterio del 90 percentil, sitúa valores de presión en $90 \mathrm{~mm} / \mathrm{Hg}$ para la diastólica y 
Tabla 6

Análisis de tendencia del estado de hiperreactividad vascular según la edad. $\mathrm{N}=267$. Rodas, 1998

\begin{tabular}{|c|c|c|c|c|c|c|}
\hline \multirow{2}{*}{ Grupo de edades } & \multicolumn{2}{|c|}{ Hiperreactivos } & \multicolumn{2}{|c|}{ Normotensos } & $O R$ & IC $95 \%$ \\
\hline & No & $\%$ & No & $\%$ & & \\
\hline $18-29$ & 13 & 12.4 & 92 & 87.6 & & \\
\hline $30-39$ & 8 & 14.5 & 47 & 85.5 & 1.20 & $0.47,3.11$ \\
\hline $40-49$ & 14 & 30.4 & 32 & 69.6 & 3.10 & $1.23,7.28$ \\
\hline $50-59$ & 9 & 28.1 & 23 & 71.9 & 2.77 & $1.06,7.27$ \\
\hline 60 y más & 9 & 30 & 21 & 70 & 3.03 & $1.15,8.03$ \\
\hline
\end{tabular}

Tabla 7

Número y por ciento de hiperreactivos y normorreactivos vasculares en dependencia de la ingestión de bebidas alcohólicas. $\mathrm{N}=267$. Rodas, 1998

\begin{tabular}{|c|c|c|c|c|c|}
\hline \multirow{2}{*}{ Categoria } & \multicolumn{2}{|c|}{ Hiperreactivos } & \multicolumn{2}{|c|}{ Normorreactivos } & \multirow{2}{*}{ Total } \\
\hline & $N$ & $\%$ & $N$ & $\%$ & \\
\hline Nunca ocasional & 27 & 19.3 & 113 & 80.7 & 140 \\
\hline Moderado & 17 & 19.5 & 70 & 80.5 & 87 \\
\hline Excesivo & 14 & 35.0 & 26 & 65.0 & 40 \\
\hline Total & 58 & 21.7 & 209 & 78.3 & 267 \\
\hline
\end{tabular}

$140 \mathrm{~mm} / \mathrm{Hg}$ para la sistólica. Este criterio aumenta el rango en el que se considera a un individuo como $\mathrm{HRCV}$, cuando en estado de reposo tiene niveles de presión normales, lo que pudiera contribuir de forma significativa a mejorar la prevención de la HTA y de las enfermedades cardiovasculares.

Se ha demostrado que la relación entre HF y HTA parece estar dada por una acentuación de la actividad del sistema nervioso simpático, que provoca un aumento del gasto cardiaco, frecuencia cardiaca, contractilidad miocardica y resistencia periférica total ${ }^{10.11}$. Girdler SS y otros ${ }^{11}$ encontraron una mayor respuesta cardiovascular en mujeres fumadoras con respecto a los hombres fumadores.

A diferencia de estos resultados, no se demostró una asociación significativa entre el HF y la HRCV; tampoco se evidenciaron diferencias significativas, $p>0.05$, entre los niveles de TA de los fumadores y los no fuma- dores, estandarizados por sexo. Es probable que estos resultados se deban a que sólo se utilizaron, a diferencia de otros estudios, individuos normotensos y no toda la población; o a que, simplemente, en estas personas los niveles de reactividad cardiovascular realmente no están afectados por el hábito de fumar.

Cuando se analizan otros factores de riesgo vemos que, la edad, los APF de HTA y el sedentarismo, presentan una asociación significativa $\mathrm{P}<0.05$, con el estado de RCV. La edad estuvo asociada a partir de los 40 años, donde el porcentaje de individuos que presentó esta situación rondó el $30 \%$, y el riesgo fue alrededor de 3 veces mayor respecto a los individuos con menos de 30 años. En la literatura encontramos varios trabajos que involucran la edad con los niveles de TA 12.13; otros autores no han encontrado asociación entre la edad y el aumento de los niveles de TA con pruebas de ejercicio isométrico en individuos con HTA esencial 
ligera, pero sí en los que tienen HTA severa $^{12}$.

Se ha visto asociación entre el nivel de obesidad y la hiperreactividad cardiovascular. Tulio $\mathrm{S}^{13}$, observó que los adolescentes obesos e hipertensos desarrollaban un incremento significativo de la presión ante el ejercicio físico, con respecto a los adolescentes hipertensos pero no obesos. Por tanto, conociendo la relación entre sedentarismo y obesidad es posible explicar que en los individuos sedentarios el riesgo de $\mathrm{HRCV}$ sea significativamente mayor que en los que practican actividad física.

Lemne $\mathrm{CE}^{14}$, encontró que los niños con APF de HTA, tenían niveles de TA ante el estrés significativamente superiores al resto de los niños. Esto evidencia lo descrito por otros autores que asocian ambas variables. El riesgo de HRCV con la PPS fue significativamente superior en las personas con APF de HTA, siendo casi 4 veces mayor $\mathrm{OR}=$ $3,85^{2,7,19}$.

No se encontraron diferencias significativas entre la IBA y la HRCV. Sí es importantc señalar que el $35 \%$ de los individuos que ingerían bebidas alcohólicas habitualmente o excesivamente eran clasificados como HRCV, por sólo un 19\% del resto de los grupos. Este elemento refleja que, independientemente de que no haya diferencias significativas entre los grupos, existe un mayor riesgo de $\mathrm{HRCV}$ en los individuos que consumen alcohol abundantemente, y si no es significativa esta diferencia, pudiera estar dada por el pequeño número de personas que se incluyen en esta categoria.

Como conclusiones podemos decir que, existe una asociación entre diferentes FRCV y el incremento de la TA en individuos normotensos cuando se someten a estrés físico. Esta situación es evidente en los individuos que tienen APF de HTA, en los sedentarios $y$ en los que tienen edad igual o mayor a 40 años.
El HF y la IBA no se asoció significativamente a la HRCV en esta población de estudio, pero no obstante no se puede dejar de tener en cuenta la conocida vinculación que tienen estos dos factores con la HTA y las enfermedades cardiovasculares.

\section{BIBLIOGRAFÍA}

1. Majahalme S, Turjanmaa V, Tuomisto M, Lu H, Uusitalo A. Blood pressure responses to exercise as predictors of blood pressure level after 5 years. Am J Hypertens 1997 Jan; 10(1):106-16.

2. Falner B. The role of cardiovascular reactivity as a mediator of hypertesion in African Americans. Sem;Nphrol 1996 Mar; 16 (2):117-25.

3. Wennerberg SR, Schncider RH, Waloton KG, Maclean CR, Levitsky DK, Salermo JW, at al. A controlled study of the effects of the trancendental meditation progrom on cardiovascular reactivity and ambulatory blood pressure. Int $\mathrm{J}$ Neurosci 1997 Jan; 89(1-2):15-28.

4. Kral BG, Becker LC, Blumenthal RS, Akersano $\mathrm{T}$, Fleisher $\mathrm{L} \Lambda$, Yook RM, et al. Exaggerated reactivity to mental stress is associated with exercise induced myocardic ischemia in an asyntomatic hig-risk population. Circulation $1997 \mathrm{Dec}$; 96(12): 4246- 53 .

5. Wilson MF, Sung BH, Pincob GA, Lovallo WR. Exaggerated pressure responsed to exercise in men at risk for systemic hypertension. Am. J Cardiol 1990 sep 15; 66(7):731-6.

6. Treiber FA, Strong WB, Arensman FW, Forrest T, Davis H, Musante L. Family history of myocardial in faretion and hemodinamic responces to exercise in young blackboys. Am J Dis Child 1991 Sep; 145(9):1029-33.

7. Paz BH. Valor de la ergotric para el diagnostico precoz de la HTA. En: Hiram Paz Basanta. Algunas consideraciones fisiológicas para el diagnóstico precoz y profilaxis de la hipertension arterial. Villa Clara: Instituto Superior de Ciencias Médicas; 1991.p. 14-20.

8. Cantor A. Lid N Use of Isometric exercise testing replace. Isotonic testing of detect and evalualed hipertensive populations. Am $J$ Cardiol 68.665-666, 1991 .

9. Liga Española contra la Hipertensión Arterial. Hipertensión Arterial y Arteriosclerosis. Madrid; 1995.p.57. 
10. Winniford MD. Smoking and cardiovascular function. J Hypertens Suppl, 1990 Sep, 8:5, S17-23.

11. Girdler SS; Jamner LD; Jarvik M; Soles JR; Shapiro D. Smoking status and nicotine administration differentially modify hemodynamic stress reactivity in men and women. Psychosom Med 1997 May; 59(3): 294-306.

12. Sumimoto T; Hamada M; Muneta S; Shigematsu Y; Fujiwara Y; Sekiya M; Kazatani Y; Hiwada K.
Influence of age and severity of hypertension on blood pressure response to isometric handgrip exercise. J Hum Hypertens 1991 Oct; 5 (5):399-403.

13. Tulio S; Eglé S; Greily B. Blood pressurc response to exercise of obese and lean hypertensive and normotensive male adolescents. J Hum Hypertens, 1995 Dec; 9 (12): 953-8.

14. Lemne CE. Increased blood pressure reactivity in children of borderline hypertensive fathers. J Hypertens 1998 Sep; 16(9):1243-8. 